\title{
THE FITNESS CONSEQUENCES OF MULTIPLE-LOCUS HETEROZYGOSITY UNDER THE MULTIPLICATIVE OVERDOMINANCE AND INBREEDING DEPRESSION MODELS
}

\author{
Peter E. SMouse \\ Department of Human Genetics, University of Michigan, Ann Arbor, MI 48109
}

\begin{abstract}
There is a growing body of literature suggesting that the fitness of an individual increases with the observed number of heterozygous loci. Broad theoretical considerations indicate that under various sorts of balancing selection, this is what one should generally expect in a population of multiple-locus genotypes. To date, however, it has not been possible to distinguish between two potential explanations of the phenomenon. The first explanation is that the loci examined are themselves responsible for the fitness differences observed (or, equivalently, are very closely linked to those that do). The genetic variation in question is thought to be maintained in polymorphic equilibrium by some form of balancing selection. The second explanation assumes that the observed loci are themselves selectively irrelevant but that their heterozygosity reflects that of the total genome. Genomic heterozygosity is thought to be predictive of fitness, being an obverse measure of generalized inbreeding depression. We provide a formal derivation of an explicit relationship between fitness and multiple-locus genotype for a simple form of the first explanation, the multiplicative overdominance model. The inbreeding depression model is a degenerate special case of this more general formulation. A formal estimation and testing framework is constructed that should facilitate evaluation of the two models with empiric data on heterozygosity and fitness.
\end{abstract}

Received June 7, 1985. Accepted June 3, 1986

The accumulating allozyme data from natural populations have established convincingly that genetic variation is widespread in a great many organisms, but we still have no compelling evidence to suggest how much of that variation is maintained by natural selection. Quite a bit of effort has been expended on the search for meaningful selective differences among alleles (or genotypes) at particular loci, with at least modest success (e.g., Tsakas and Krimbas, 1970; Vigue and Johnson, 1973; Cavener and Clegg, 1981 $a, 1981 b$; DiMichele and Powers, 1982; Hilbish et al., 1982; Oakeshott et al., 1982; Burton and Feldman, 1983; Watt, 1983; Watt et al., 1983), but the vast majority of segregating loci in most of the species surveyed so far have never been examined in enough detail to characterize selective factors in satisfactory detail. Indeed, the effort required to "prove" the selective relevance of even a single locus in even a single species is so prohibitive that we can never hope to do so for more than a small fraction of the locus/species combinations of interest. The careful effort to evaluate particular cases has been rewarding in a variety of ways, and is to be encouraged, but exhaustive enumeration is neither an attractive nor a feasible route to wide generalization about the maintenance of observed genetic variability; some other approach is needed.

Single-locus analysis of the relation between genetic variation and fitness has not been very rewarding, and a number of workers have been led to consider the impact of multiple-locus genotypes on fitness. In particular, several authors have attempted to correlate fitness or some surrogate measure with the number of heterozygous loci in an individual. Some workers find an increase in fitness with increasing heterozygosity (Schall and Levin, 1976; Singh and Zouros, 1978; Bottini et al., 1979; Zouros et al., 1980; Mitton et al., 1981; Koehn and Shumway, 1982; Pierce and Mitton, 1982; Ledig et al., 1983; Leary et al., 1984), but others do not (e.g., Mukai et al., 1974; Gaines et al., 1978; Knowles and Grant, 1981; Mitton et al., 1981).

Turelli and Ginzburg (1983) have theoretically demonstrated that if allozyme polymorphisms are maintained by any of several forms of balancing selection, then we should observe a general increase in fitness with an increase in the number of heterozygous loci. Whether the variation in 
question is actually maintained by balancing selection remains to be seen, of course, but the observations are generally in agreement with predictions based on a broad concept of balancing selection.

Alternatively, it has been suggested that measured heterozygosity may merely reflect overall genomic heterozygosity and that highly homozygous genotypes suffer from inbreeding depression (Ledig et al., 1983). Mitton and Pierce (1980) and Chakraborty (1981) have shown, however, that heterozygosity from a small sample of loci is a poor indicator of total genomic heterozygosity for an individual, suggesting that there should be little or no association if inbreeding depression is the root cause of the phenomenon. On the other hand, inbreeding depression can be a powerful force (cf. Franklin, 1970), so even a small correlation between observed and genomic heterozygosity might be sufficient to account for the loose association observed between heterozygosity and fitness.

It is difficult to choose between these alternative explanations, because our current assessment procedures are almost hopelessly crude; they are not designed to distinguish between the two selective hypotheses. In current practice, no attention is paid to which loci are heterozygous, and most of the potentially useful information is suppressed in the interest of convenient summarization. The objective of this paper is to develop a predictive model for the relationship between fitness and the particular multiple-locus genetic state of a single individual. The model employed is the simplest I could use, the multiplicative overdominance model for unlinked loci. I present an evaluation procedure: (1) that is very easily implemented, (2) that provides a test of the null hypothesis of no selective differences among observed genotypes, (3) that distinguishes between genomic inbreeding and specific-locus effects, (4) that permits a determination of the marginal effect of each locus, and (5) that facilitates an assessment of the multiplicative overdominance assumption.

I begin with a consideration of the pattern of fitness to be expected from a set of twoallele loci, then describe the pattern to be expected of multiple-allele loci, and then present an assessment procedure for evaluating the hypothesis. I argue that the inbreeding depression hypothesis is a degenerate special case of the more general formulation. In a later paper (Bush et al., unpubl.), the predictions of the two models are compared for a series of population samples from pitch pine (Pinus rigida Mill.).

\section{A Set of Two-Allele Loci}

Consider a diploid population with $K$ segregating loci, unlinked and with two alleles each. I assume that the two alleles at each locus are codominant, as is usual with allozyme electromorphs; denote them as $A_{1}$ and $A_{2}, B_{1}$ and $B_{2}$, and their allele frequencies as $P_{\mathrm{A}}$ and $Q_{\mathrm{A}}, P_{\mathrm{B}}$ and $Q_{\mathrm{B}}$, etc. I presume that the population has already attained multiple-locus Hardy-Weinberg equilibrium.

I envisage a broad sort of balancing selection that maintains the genetic variation in equilibrium. The marginal fitness values for any one locus are functions of genotypic frequencies and of the fitness values of the multiple-locus genotypes. I postulate that at equilibrium the net balance of forces is such that the marginal fitness array at each locus shows overdominance. Karlin and Liberman $(1979 a, 1979 b)$ have shown that for either additive or multiplicative fitness across a set of "loosely linked" loci, stable polymorphic equilibria will exhibit marginal overdominance. More general fitness schemes leading to multiple-locus stable polymorphism are not guaranteed to yield marginal overdominance at equilibrium (Lewontin and Kojima, 1960; Ewens and Thomson, 1977; Hastings, 1981, 1982), but Turelli and Ginzburg (1983) have shown that marginal overdominance is the most frequent consequence of multiple-locus polymorphism. For purposes of exposition, then, let the $A$-locus be represented as shown in the panel below.

\begin{tabular}{llll}
\hline Genotype & $A_{1} A_{1}$ & $A_{1} A_{2}$ & $A_{2} A_{2}$ \\
Frequency & $P_{\mathrm{A}}{ }^{2}$ & $2 P_{\mathrm{A}} Q_{\mathrm{A}}$ & $Q_{\mathrm{A}}{ }^{2}$ \\
Fitness $\left(W_{\mathrm{A}}\right)$ & $\mathrm{e}^{-S_{\mathrm{A}}}$ & 1 & $\mathrm{e}^{-T_{\mathrm{A}}}$ \\
\hline
\end{tabular}

The values of $S_{\mathrm{A}}$ and $T_{\mathrm{A}}$ are assumed to be small and positive, compatible with the notion that marginal selective differentials are small. It will be convenient to use the fact 
that $\left(1-S_{\mathrm{A}}\right) \approx \exp \left\{-S_{\mathrm{A}}\right\}$ and $\left(1-T_{\mathrm{A}}\right) \approx$ $\exp \left\{-T_{\mathrm{A}}\right\}$ for small $S_{\mathrm{A}}$ and $T_{\mathrm{A}}$, and I shall switch back and forth between these notational forms whenever convenient.

I have assumed Hardy-Weinberg frequencies for expository purposes, and this warrants some comment. Not all types of heterotic selection lead to Hardy-Weinberg proportions at equilibrium, but where selective differentials are small, departures from Hardy-Weinberg proportions will be small as well. Moreover, there is little evidence in natural populations for large departures from Hardy-Weinberg proportions that could be attributed to large selective differentials, although the difficulties of detecting departures from Hardy-Weinberg proportions are well known (e.g., Lewontin and Cockerham, 1959; Ward and Sing, 1970; Chakraborty and Rao, 1972; Haber, 1980). Given the observations to date, the assumption of Hardy-Weinberg proportions at equilibrium is a reasonable first approximation.

Using the fact that $\left(1-S_{\mathrm{A}}\right) \approx \exp \left\{-S_{\mathrm{A}}\right\}$ and $\left(1-T_{\mathrm{A}}\right) \approx \exp \left\{-T_{\mathrm{A}}\right\}$ for small $S_{\mathrm{A}}$ and $T_{\mathrm{A}}$, standard theory (e.g., Li, 1978) yields the following equilibrium frequencies for the $A$-locus:

$$
P_{\mathrm{A}}=\frac{T_{\mathrm{A}}}{S_{\mathrm{A}}+T_{\mathrm{A}}} \text { and } Q_{\mathrm{A}}=\frac{S_{\mathrm{A}}}{S_{\mathrm{A}}+T_{\mathrm{A}}} .
$$

The more fit of the two homozygotes is the more common. The central idea in what follows is that some homozygotes are more fit than others. Rather than using the number of homozygous loci as a criterion of fitness, I construct testable hypotheses about the differences of homozygote fitness values from the observed allele frequencies. To do that, I construct a measure of the "adaptive distance" between any homozygote and the (heterozygous) optimum genotype. The least fit homozygote should be furthest from this optimum. The scaling of adaptive distance is also helpful in determining the relative fitness impact of selection at different loci. Consider the quantity $\left(X_{\mathrm{A}}\right)$ as a candidate measure of adaptive distance for $A$-locus genotypes.

\begin{tabular}{llll}
\hline Genotype & $A_{1} A_{1}$ & $A_{1} A_{2}$ & $A_{2} A_{2}$ \\
Fitness $\left(W_{\mathrm{A}}\right)$ & $\mathrm{e}^{-S_{\mathrm{A}}}$ & 1 & $\mathrm{e}^{-T_{\mathrm{A}}}$ \\
Distance $\left(X_{\mathrm{A}}\right)$ & $P_{\mathrm{A}}^{-1}$ & 0 & $Q_{\mathrm{A}}^{-1}$ \\
\hline
\end{tabular}

The fitness values $\left(W_{\mathrm{A}}\right)$ above are marginal genotypic averages, of course, and we really desire values for individuals. For the $j$ th individual, the natural logarithm of fitness (denoted $\left.Y_{\mathrm{A}}\right)$ and adaptive distance $\left(X_{\mathrm{A}}\right)$ are simply related:

$$
\begin{aligned}
Y_{\mathrm{A}}\left(11_{j}\right) & =\log \left[W_{\mathrm{A}}\left(11_{j}\right)\right] \\
& =-S_{\mathrm{A}}+\epsilon_{j} \\
& =-\alpha \cdot X_{\mathrm{A}}(11)+\epsilon_{j} \\
& =\left[-\alpha / P_{\mathrm{A}}\right]+\epsilon_{j} \\
Y_{\mathrm{A}}\left(12_{j}\right) & =\log \left[W_{\mathrm{A}}\left(12_{j}\right)\right] \\
& =-\alpha \cdot X_{\mathrm{A}}(12)+\epsilon_{j} \\
& =\epsilon_{j} \\
Y_{\mathrm{A}}\left(22_{j}\right) & =\log \left[W_{\mathrm{A}}\left(22_{j}\right)\right] \\
& =-T_{\mathrm{A}}+\epsilon_{j} \\
& =-\alpha \cdot X_{\mathrm{A}}(22)+\epsilon_{j} \\
& =\left[-\alpha / Q_{\mathrm{A}}\right]+\epsilon_{j},
\end{aligned}
$$

where the error terms $\left(\epsilon_{j}\right)$ reflect the fact that individuals have fitness (or surrogate) values departing from the genotypic averages, due to differences in genotype at other loci, due to random environmental influences, measurement error, and so on. These error terms (deviations from genotypic expectations) are assumed to be independent and identically distributed, with mean $(\bar{\epsilon})=0$ and variance $\left(\sigma_{\epsilon}^{2}\right)$. The slope coefficient $(\alpha)$ takes the value:

$$
\alpha=\frac{S_{\mathrm{A}} T_{\mathrm{A}}}{S_{\mathrm{A}}+T_{\mathrm{A}}} .
$$

The term $\alpha$ is traditionally referred to as the "segregational genetic load" for the $A$-locus (Morton et al., 1956). Whether one views genetic load as the cost of heterosis or as a measure of lost opportunity resulting from the impossibility of maintaining a pure population of the optimal (heterozygous) genotype, it is a measure of the magnitude of differential selection operating on genotypes at the locus. The relationship between $\log$ (fitness) and adaptive distance is linear, and the slope of the line is the intensity of selection for the locus. For scaling the asymmetry between $X_{\mathrm{A}}(11)$ and $X_{\mathrm{A}}(22), \alpha$ is of no concern, since:

$$
\frac{\left[-\alpha / P_{\mathrm{A}}\right]}{\left[-\alpha / Q_{\mathrm{A}}\right]}=\frac{S_{\mathrm{A}}}{T_{\mathrm{A}}} .
$$




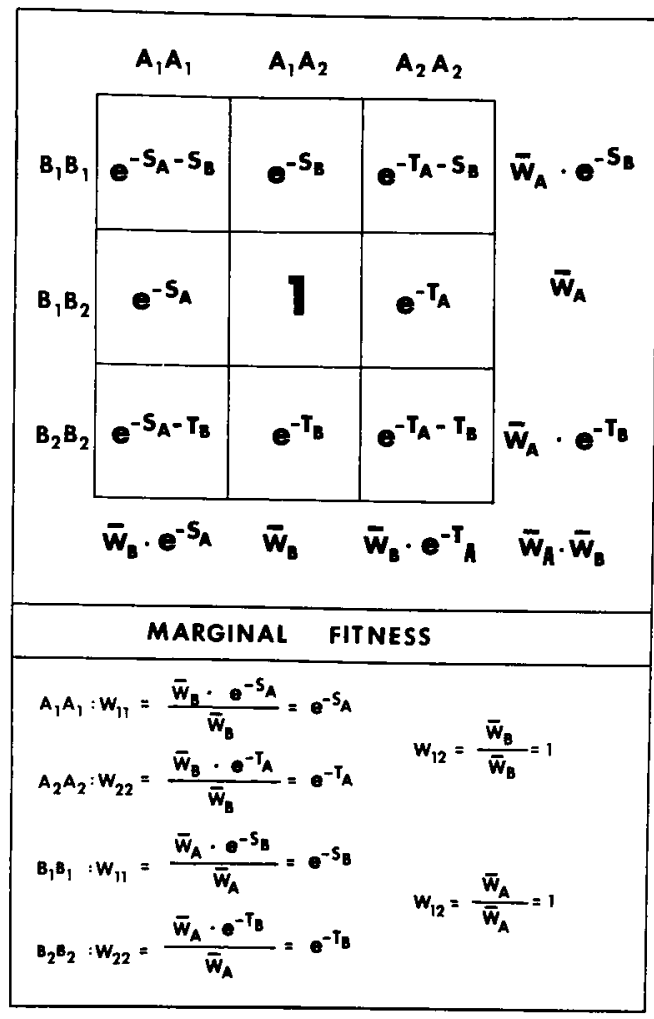

FIG. 1. Two-locus fitness values for the multiplicative overdominance model, with marginal relative fitness values for each locus.

Now, consider a second locus, also marginally overdominant. Genotypic frequencies are given (at least approximately) by two-locus Hardy-Weinberg expectations at equilibrium. Having assumed multiplicative fitness across a set of unlinked loci, there should be no gametic disequilibria between the $A$ and $B$ loci at equilibrium (Karlin and Liberman, 1979a, 1979b). The adaptive distances of $B_{1} B_{1}, B_{1} B_{2}$, and $B_{2} B_{2}$ are $X_{\mathrm{B}}(11)=P_{\mathrm{B}}^{-1}, X_{\mathrm{B}}(12)=0$, and $X_{\mathrm{B}}(22)=$ $Q_{\mathrm{B}}{ }^{-1}$, respectively. In keeping with the multiplicative overdominance model, the twolocus fitness array is as shown in Figure 1. Each double homozygote (corner) has lower fitness than either flanking single-locus homozygote, and each single-locus homozygote has lower fitness than the two-locus heterozygote (center). Fitness decreases when additional loci are homozygous, but the decreases are not identical for all genotypes with the same level of homozygosity, a fact I make use of below.
Since the fitness values are assumed to be multiplicative across loci, and since there are no disequilibria, the natural logarithm of fitness can be represented by (cf. Appendix):

$$
Y_{j}=\log \left(W_{j}\right)=-\alpha \cdot X_{\mathrm{A}}-\beta \cdot X_{\mathrm{B}}+\epsilon_{j},
$$

where the slope coefficients are:

$$
\alpha=\frac{S_{\mathrm{A}} T_{\mathrm{A}}}{S_{\mathrm{A}}+T_{\mathrm{A}}} \text { and } \beta=\frac{S_{\mathrm{B}} T_{\mathrm{B}}}{S_{\mathrm{B}}+T_{\mathrm{B}}},
$$

the respective intensities of selection for the two loci. Allele frequencies are sufficient to gauge the asymmetry of adaptive distance within a locus, but the respective intensities are needed to weight the loci. Note, for example, that both the parameter sets $\{S=$ $0.01, T=0.02\}$ and $\{S=0.05, T=0.10\}$ imply $\{P=2 / 3, Q=1 / 3\}$, but they represent rather different intensities of selection. The allele frequencies are observable, but it is necessary to estimate the slopes. There are nine genotypes, each with a different adaptive distance. To see what this means, consider the following example; let $S_{\mathrm{A}}=0.02$, $T_{\mathrm{A}}=0.04, S_{\mathrm{B}}=0.015$, and $T_{\mathrm{B}}=0.045$. The equilibrium allele frequencies are $P_{\mathrm{A}}=2 / 3$, $Q_{\mathrm{A}}=1 / 3, P_{\mathrm{B}}=3 / 4$, and $Q_{\mathrm{B}}=1 / 4$, and the slopes (intensities) are $\alpha=0.0133$ and $\beta=0.0113$.

A plot of the logarithm of fitness against two-locus adaptive distances is presented in Figure 2a, where the $X$-values for the $A$ and $B$ loci are weighted by $\alpha$ and $\beta$, respectively. The optimal genotype $\left(A_{1} A_{2} B_{1} B_{2}\right)$ is at one end of the scale and the worst $\left(A_{2} A_{2} B_{2} B_{2}\right)$ is at the other end, but there is no strict order of fitness with the number of heterozygous loci. For example, $A_{1} A_{1} B_{1} B_{1}$ has higher fitness than either $A_{1} A_{2} B_{2} B_{2}$ or $A_{2} A_{2} B_{1} B_{2}$. An analogue of the more usual treatment is presented in Figure $2 b$, where $\log$ (fitness) is plotted against the number of homozygous loci. The usual monotonicity is evident, but this presentation is less informative than that of Figure 2a. Moreover, since the points of the graph are averages for two-locus genotypes, there is individualto-individual variation around the regression line, more variation in Figure $2 b$ than in Figure 2a.

The extension to multiple loci, each with two alleles, is obvious. Given the same sort of adaptive distance measures, the predictive model becomes: 


$$
\begin{aligned}
Y_{j}= & \log \left(W_{j}\right) \\
= & -\alpha \cdot X_{\mathrm{A}}-\beta \cdot X_{\mathrm{B}}-\ldots \\
& -\kappa \cdot X_{\mathrm{K}}+\epsilon_{j},
\end{aligned}
$$

where the slope coefficients are the respective intensities of selection for the various loci. The model encompassed by Equation (7) is richer in predictive detail than the standard treatment; with $K$ loci, the usual treatment allows for only $(K+1)$ heterozygosity classes. With the same $K$ loci, there are almost always more than $(K+1)$ different genotypes in the adaptive distance model, sometimes substantially more.

\section{A Multiple-Allele Locus}

The mathematical theory for the selective maintenance of multiple-alleles in polymorphic equilibrium is considerably more complicated than is that for a pair of alleles. The conditions that must be satisfied are very demanding, and simple heterosis is neither necessary nor sufficient (e.g., Mandel, 1970; Lewontin et al., 1978; Li, 1978). However, provided that a state of balanced equilibrium is assumed to exist, there are certain conditions that will be satisfied. Consider the three-allele $C$-locus, which can be represented as follows.

Genotype $\quad \begin{array}{llllll}C_{1} C_{1} & C_{1} C_{2} & C_{1} C_{3} & C_{2} C_{2} & C_{2} C_{3} & C_{3} C_{3}\end{array}$ $\begin{array}{llllllll}\text { Frequency } & P_{\mathrm{C}}{ }^{2} & 2 P_{\mathrm{c}} Q_{\mathrm{C}} & 2 P_{\mathrm{C}} R_{\mathrm{C}} & Q_{\mathrm{c}}{ }^{2} & 2 Q_{\mathrm{C}} R_{\mathrm{C}} & R_{\mathrm{C}}{ }^{2}\end{array}$ $\begin{array}{lllllll}\text { Fitness } & W_{11} & W_{12} & W_{13} & W_{22} & W_{23} & W_{33}\end{array}$

Now, pool $C_{2}$ and $C_{3}$ into $C_{1^{\prime}}$, reducing the locus to a pair of allelic classes $\left(C_{1}\right.$ and $\left.C_{1}\right)$.

\begin{tabular}{llll}
\hline Genotype & $C_{1} C_{1}$ & $C_{1} C_{1^{\prime}}$ & $C_{1^{\prime}} C_{1^{\prime}}$ \\
Frequency & $P_{\mathrm{C}^{2}}$ & $2 P_{\mathrm{C}}\left(1-P_{\mathrm{C}}\right)$ & $\left(1-P_{\mathrm{C}}\right)^{2}$ \\
Fitness & $W_{11}$ & $W_{11}$ & $W_{1^{\prime} \mathrm{I}^{\prime}}$ \\
\hline
\end{tabular}

The fitnesses of the synthetic genotypes $\left(W_{11}\right.$ and $\left.W_{1,1}\right)$ are:

$$
\begin{aligned}
W_{11^{\prime}} & =\frac{\left(2 P_{\mathrm{C}} Q_{\mathrm{C}} W_{12}+2 P_{\mathrm{C}} R_{\mathrm{C}} W_{13}\right)}{\left(2 P_{\mathrm{C}} Q_{\mathrm{C}}+2 P_{\mathrm{C}} R_{\mathrm{C}}\right)} \\
& =\frac{\left(Q_{\mathrm{C}} W_{12}+R_{\mathrm{C}} W_{13}\right)}{\left(1-P_{\mathrm{C}}\right)} \\
W_{1^{\prime} 1^{\prime}} & =\frac{\left(Q_{\mathrm{C}}^{2} W_{22}+2 Q_{\mathrm{C}} R_{\mathrm{C}} W_{23}+R_{\mathrm{C}}^{2} W_{33}\right)}{\left(1-P_{\mathrm{C}}\right)^{2}} .
\end{aligned}
$$

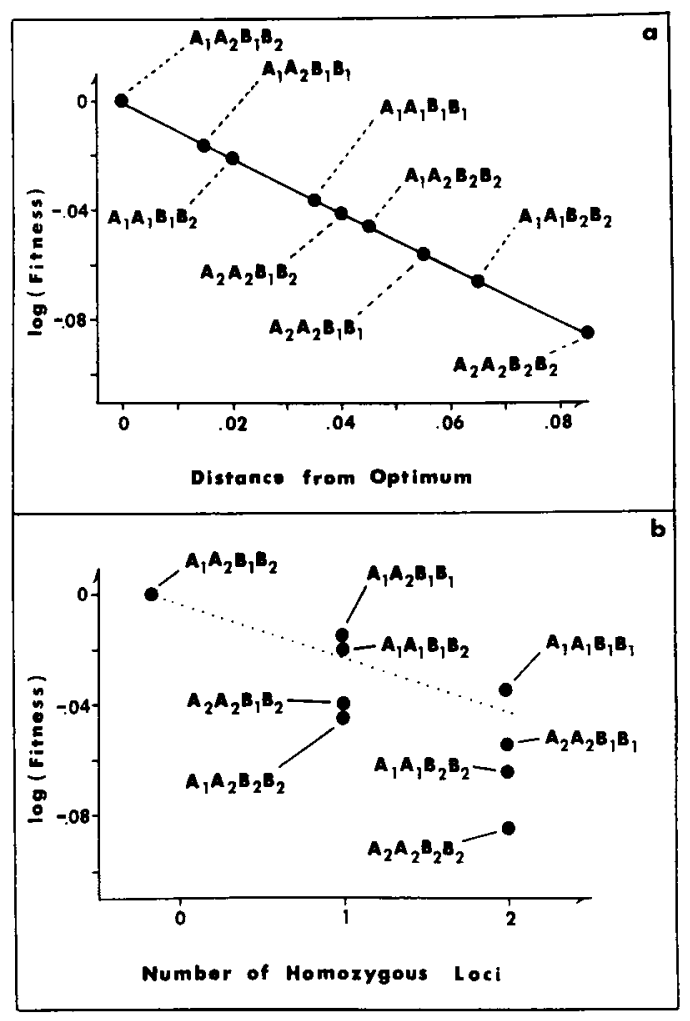

FIG. 2. An example of the relationship between $\log$ (fitness) and genotype for two loci: a) according to the adaptive distance formulation, and b) reflected in the number of homozygous loci.

Alternatively, one might pool $C_{1}$ and $C_{3}$ into $C_{2}$, reducing the locus to a different pair of allelic classes, or even $C_{1}$ and $C_{2}$ into $C_{3}$, reducing the locus to a third twoallele form. For each of these alternative lumpings, it is straightforward to define synthetic fitness measures along the lines indicated by Equations (8a) and (8b). A necessary condition for (consequence of) a stable polymorphic equilibrium is that:

$$
W_{11}, W_{1^{\prime} 1}<W_{11}
$$

and

$$
W_{22}, W_{2^{\prime} 2^{\prime}}<W_{22^{\prime}}
$$

and

$$
W_{33}, W_{3^{\prime} 3^{\prime}}<W_{33^{\prime}}
$$

To treat a three-allele locus, it is only necessary to pool two of the alleles into an "other alleles" class, and then use the two-allele treatment, merely adding the locus to the 


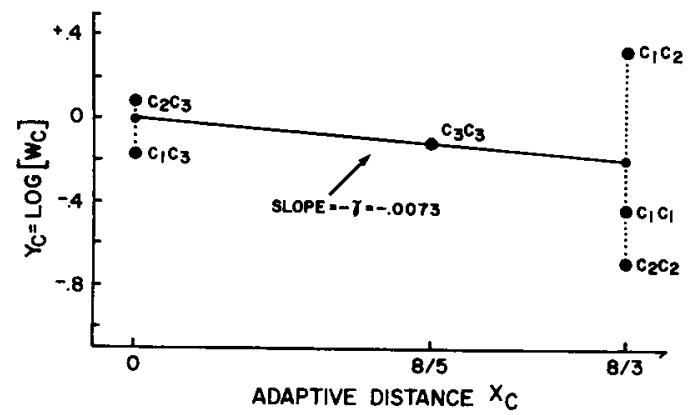

Fig. 3. An example of the relationship between $\log$ (fitness) and genotype for a three-allele locus. The line represents the model specification, which is an exact representation if $C_{1} C_{1}, C_{1} C_{2}$, and $C_{2} C_{2}$ are pooled into $C_{3}, C_{3}$, and if $C_{1} C_{3}$ and $C_{2} C_{3}$ are pooled into $C_{3} C_{3^{\prime}}$. The actual $\log$ (fitnesses) of all six genotypes are also plotted.

array in (7). The only question is which of the three available pooling strategies to use. The best strategy is to use the most frequent allele as $C_{1}$, lumping the other two into $C_{1}$. The major reason for this is that evaluation of the model will be most effective if the rarer of the two homozygotes is present in the sample in nontrivial frequencies. Pooling of the rarer alleles should generally ensure this.

To illustrate the three-allele analysis further, I envision a three-allele $C$-locus with a frequency/fitness specification slightly modified from an example by Li (1978 p. 44).

\begin{tabular}{lcccccc} 
Genotype & $C_{1} C_{1}$ & $C_{1} C_{2}$ & $C_{1} C_{3}$ & $C_{2} C_{2}$ & $C_{2} C_{3}$ & $C_{3} C_{3}$ \\
Frequency & $\frac{1}{64}$ & $\frac{4}{64}$ & $\frac{10}{64}$ & $\frac{4}{64}$ & $\frac{20}{64}$ & $\frac{25}{64}$ \\
Fitness & 0.925 & 1.000 & 0.950 & 0.900 & 0.975 & 0.955 \\
\hline
\end{tabular}

The genotypic frequencies are determined by the fitness values and are computed as in $\mathrm{Li}(1978)$ :

$$
\begin{aligned}
& \tilde{P}_{\mathrm{C}}=0.125 \\
& \tilde{Q}_{\mathrm{C}}=0.250 \\
& \tilde{R}_{\mathrm{C}}=0.625 .
\end{aligned}
$$

These equilibrium frequencies and fitness values imply frequencies and fitness values for the synthetic genotypes $\left(C_{1} C_{1}\right.$, and $\left.C_{1}, C_{1}\right),\left(C_{2} C_{2}\right.$, and $\left.C_{2}, C_{2}\right),\left(C_{3} C_{3^{\prime}}\right.$ and $\left.C_{3^{\prime}} C_{3^{\prime}}\right)$, and these latter satisfy, as they must, the conditions required for a stable polymorphism:

$$
\begin{gathered}
W_{11}=0.9250, \quad W_{1^{\prime} 1^{\prime}}=0.9587 \\
<W_{11^{\prime}}=0.9643 \\
W_{22}=0.9000, \quad W_{2^{\prime} 2^{\prime}}=0.9527 \\
<W_{22^{\prime}}=0.9792 \\
W_{33}=0.9550, \quad W_{3^{\prime} 3^{\prime}}=0.9472 \\
<W_{33^{\prime}}=0.9667
\end{gathered}
$$

Since $C_{3}$ is the most frequent allele, lump $C_{1}$ and $C_{2}$ into $C_{3}$, and construct the following $(X, Y)$-specification for the $C$-locus.

\begin{tabular}{lccccc}
$\begin{array}{l}\text { Genotype } \\
C_{1} C_{1}\end{array}$ & $C_{1} C_{2}$ & $C_{1} C_{3}$ & $C_{2} C_{2}$ & $C_{2} C_{3}$ & $C_{3} C_{3}$ \\
$\hat{Y}_{\mathrm{C}}$ & & & & & \\
-0.0195 & -0.0195 & 0 & -0.0195 & 0 & -0.0117 \\
$X_{\mathrm{C}}$ & & & & & \\
$\quad 2.6667$ & 2.6667 & 0 & 2.6667 & 0 & 1.6000 \\
$\log \left(W_{\mathrm{C}}\right)$ & & & & & \\
-0.0426 & +0.0328 & -0.0168 & -0.0690 & +0.0083 & -0.0117 \\
\hline
\end{tabular}

The $\log \left(W_{\mathrm{C}}\right)$ values are scaled relative to the average fitness of $C_{1} C_{3}$ and $C_{2} C_{3}(0.9667)$. A plot of $\log \left(W_{\mathrm{C}}\right)$ against $X_{\mathrm{C}}$ is presented in Figure 3. The theoretical line $\left(\hat{Y}_{\mathrm{C}}\right)$ is that predicted by the model (with $C_{1}$ and $C_{2}$ pooled); the slope is $-\gamma=-0.0073$. The six logarithmically transformed fitness values are plotted against their respective adaptive distance values. The weighted averages of the fitness values of the genotypes having the same $X_{\mathrm{C}}$-values fit the line exactly, but individual genotypes do not. This is an unavoidable consequence of pooling the least common alleles. There is always some loss of information with pooling, but what information remains is valid.

The extension of this treatment to multiple-allele loci is straightforward. Stable polymorphic equilibrium for multiple alleles implies overdominance for all of the synthetic pairs:

$$
W_{i i}, W_{i^{\prime} i^{\prime}}<W_{i i^{\prime}} \text { for all alleles (i). (12) }
$$

All but the most common allele can be lumped into a single "other alleles" class, and the locus can be treated in two-allele form.

\section{An Estimation and Testing Framework}

The estimation of the regression coefficients (the intensities of selection) is a relatively straightforward matter that I shall develop below. Before beginning that dis- 
cussion, however, some assurance is needed that the adaptive distances ( $X$-values) are measured without error or at least with small error, relative to the error in the $Y$-values. The $X$-values are simply assigned from the allele frequencies of the population, but these frequencies are estimated with some error. Given large samples, the allele frequencies (and hence the $X$-values) will be estimated with minimal error, and it might be well in general to estimate those frequencies from a different (and hopefully larger) sample than is used to assess the relationship between heterozygosity and fitness. The real uncertainty in all of this work is the measurement of fitness (or some credible surrogate) for an individual. That task is formidable under the best of circumstances, and one can anticipate measurement and estimation errors at least an order of magnitude larger than the errors in the adaptive distances. Both problems will be discussed in more detail in a later paper (Bush et al., unpubl.). $I$ assume here that the requisite measures of fitness are in hand.

Consider a pair of two-allele loci, and recall the fitness array of Figure 1. Define a parameter vector $\theta=[\alpha, \beta]^{\prime}$. It can be shown (Appendix) that the usual regression estimator $\hat{\theta}$ is unbiased:

$$
E[\hat{\theta}]=E[\hat{\alpha}, \hat{\beta}]^{\prime}=[\alpha, \beta]^{\prime}=\theta .
$$

The model (regression) and error (residual) sums of squares are easily shown to be functions of sample size $(N)$, the error variance $\left(\sigma_{\epsilon}^{2}\right)$, and the selection intensities (Appen$\operatorname{dix})$ :

$$
\begin{aligned}
E[\mathrm{SS}(\mathrm{MODEL})]= & 2 \cdot \sigma_{\epsilon}^{2}+(N-1) \\
& \cdot\left(\alpha^{2}+\beta^{2}\right) \\
E[\mathrm{SS}(\mathrm{ERROR})]= & (N-3) \cdot \sigma_{\epsilon}{ }^{2} .
\end{aligned}
$$

Given $K$ loci, each with two alleles, the regression treatment can be extended to models involving the $K$-dimensional vector $\theta=[\alpha, \beta, \ldots, \kappa]^{\prime}$, merely by extending (13), $(14 a)$, and (14b) in the obvious fashion. Multi-allelic loci can also be treated in twoallele fashion, as I have indicated above, but it must be remembered that the error terms $\left(\epsilon_{j}\right)$ will contain variation among genotypes within a pooled class.

Since individual fitness (or surrogate) values will vary around the genotypic averages, due both to measurement error and fitness variation ascribable to unobserved loci, the observed SS(ERROR) will not be identically zero, and the regression model can be compared with observations in the usual fashion, testing the hypothesis that any subset of $\theta$ is zero, indicating a lack of selective relevance for that subset of loci. In the event that SS(ERROR) is large, it might be useful to determine whether the linear model (multiplicative overdominance) is itself adequate as a representation, since one can augment the model by adding cross-product (epistatic) terms.

One final remark on estimation and testing is in order. All of the above is predicated on the assumption that it is possible to determine relative fitness values of all $K$-locus genotypes, by reference to the optimal genotype, the $K$-locus heterozygote. In routine practice, with even a modest number of loci under examination, the $K$-locus heterozygote will frequently not be observed. In that event, some other genotype must be used as a fitness reference, which results in the addition of a nonzero intercept to (7). This convention represents a mere shift of scale, and has no consequences for the evaulation techniques presented here.

\section{The Inbreeding Depression Model}

I pointed out above that the observed relationship between heterozygosity and fitness could be the result of nonspecific inbreeding depression, with a more heterozygous genotype merely indicating a lower homozygosity level of the individual. The convention of counting the number of heterozygous loci is a special case of the adaptive distance formulation I have used here. A count of the number of heterozygous loci is tantamount to the assumption that all observed loci are equivalent in fitness terms. If the genetic loci under consideration are mere indicators of the genomic heterozygosity of the individual, then the details concerning which of the alternate allelic markers at any particular locus are homozygous will convey no useful information about the fitness of that multiple-locus genotype. In essence, that amounts to scoring all heterozygotes as $X=0$ and all homozygotes as $X=1$ and setting all regression coefficients equal. That reduces the inbreeding depression model to a degenerate special 
case of the adaptive distance model, a treatment developed by Morton et al. (1956). To evaluate that model, it is only necessary to regress $\log (W)$ on the number of homozygous loci; serious lack of fit would be an indication that the inbreeding depression model is too simple. Any significant improvement in the prediction due to fitting separate slopes for the separate loci and/or different adaptive distances for different homozygotes would be evidence that the loci under examination are important in themselves, rather than being indicators of a generalized inbreeding effect.

It is appropriate to close this section with a final comment on the inbreeding depression model. Mitton and Pierce (1980) and Chakraborty (1981) have examined the correlation between the heterozygosity/homozygosity of a subset of observed loci and that of the total genome, and have shown that the correlation is generally quite small. If the loci under examination are assumed to be of no adaptive relevance, then one might argue that the fitness effects of genomic homozygosity must be large to account for the moderate size of the necessarily indirect association between observed homozygosity and fitness. It develops, however, that this whole line of argument is in need of closer scrutiny.

Mitton and Pierce, and Chakraborty as well, employed sets of independently segregating loci. There is no correlation of homozygosity across a set of independently segregating loci, and the correlation between observed and genomic homozygosity is due solely to the fact that the former is a subset of the latter. Formally, let homozygosity at the observed loci be denoted by $(X)$ and that of all other (unobserved loci) be denoted by $\left(X^{\prime}\right)$. If genomic homozygosity is denoted by $(Z)$, then the relation between $Z$ and $X$ is merely:

$$
Z=\zeta \cdot X+(1-\zeta) \cdot X^{\prime}
$$

where $\zeta$ is the fraction of segregating loci under actual observation. The correlation between $X$ and $Z$ is thus:

$$
\begin{aligned}
r_{\mathrm{XZ}} & =\frac{\sigma_{\mathrm{XZ}}}{\sigma_{\mathrm{X}} \cdot \sigma_{\mathrm{Z}}} \\
& =\frac{\zeta \cdot \sigma_{\mathrm{X}}}{\sqrt{\zeta^{2} \cdot \sigma_{\mathrm{X}}^{2}+(1-\zeta)^{2} \cdot \sigma_{\mathrm{X}^{\prime}}}}
\end{aligned}
$$

which is nonzero because $\zeta$ is nonzero. For the inbreeding depression model, $X$ is thought to have no direct effect on fitness, so what we really need is the indirect relationship between $X$ and $Y$, through $X^{\prime}$. The relationship between $X, X^{\prime}$, and $Y$ is shown in path diagram form in Figure 4. If observed homozygosity $(X)$ has no direct effect on $Y$, then the direct path coefficient $p_{\mathrm{XY}}=$ 0 , and since $r_{\mathrm{XX}^{\prime}}=0$ as well, there is no correlation between observed homozygosity and fitness, direct or indirect (Fig. 4a). If there is an association between $X$ and $Y$, it must be due to the direct effect of $X$ on $Y$, with $p_{X Y} \neq 0$, as shown in Figure $4 \mathrm{~b}$. The basic phenomenon cannot be due to independently segregating loci that are not under observation.

The rest of the genome does not, of course, consist of only those loci segregating independently of the ones under observation. In general, closely linked loci can be expected to exhibit gametic disequilibrium with the loci under observation. In that case, $r_{\mathrm{XX}^{\prime}} \neq$ 0 , and one might very well observe an indirect correlation (associative overdominance) between $X$ and $Y$ (Kojima and Lewontin, 1969), an observable set of neutral markers that appear to be overdominant because of their disequilibria with unseen but linked markers that are under selection. More generally, the fitness-homozygosity associations observed by several authors probably represent the effects of small segments of chromosome surrounding the observed markers. Since it is not possible to distinguish between the heterozygosity of the observed markers and that of the flanking loci, the term "observed loci" might just as well be understood to mean "observed segments of chromosome."

Whether the fitness-homozygosity association is due to balancing selection or to inbreeding depression, the "loci" of interest are those under observation. There is nothing to be gained by invoking independently segregating portions of the genome that are not under observation. Inbreeding depression comes in two forms, the depression caused by deleterious recessive homozygotes at loci not supporting polymorphisms and the depression caused by homozygosity for alternate alleles at loci maintained as balanced polymorphisms (Morton et al., 
1956). Given that the "loci" under examination are those that matter, there should be no real need for the $(1,0)$ scoring system of the homozygosity treatment, and it should generally be possible to extract more information with the adaptive distance treatment.

\section{Discussion}

The adaptive distance treatment is based on the idea that marginal selective differentials are quite small; the assumption is the only one compatible with the known facts. Almost no one has been able to convincingly demonstrate large selective differentials for single loci. Moreover, the lack of large single-locus marginals makes a certain amount of theoretical sense. A marginalfitness value includes the frequency-dependent effects of all other loci, assayed or otherwise. With even a modest number of loci under consideration, no two individuals will have the same genotype, and the genetic backgrounds over which two marginal genotypes are averaged may not include a single background genotype in common. It is entirely plausible that the average fitness values of two or more random collections of genotypes will be about the same, everything else being equal. When viewing a single locus, one is merely looking at one facet of a multi-faceted genotype. Although demonstrating large marginal selective effects for single loci is bound to be difficult, the fact that several workers have detected fitness differences between groups of individuals that differ for as few as 6-12 identifiable loci (cf. Mitton and Grant, 1984) argues that the multiple-locus genome is under strong selection.

The variety of models that one might employ to generate a stable multiple-locus polymorphic array is very large. It is not possible to elaborate the detailed fitness structure of a complicated multiple-locus system from survey data alone, of course, but that is scarcely the point. An attempt to determine whether or not multiple-locus fitness is related to heterozygosity in some systematic and theoretically plausible fashion is the only real issue at stake here. Having begun with the simplest model that might do the job, multiplicative overdominance, the linearity of the relationship between

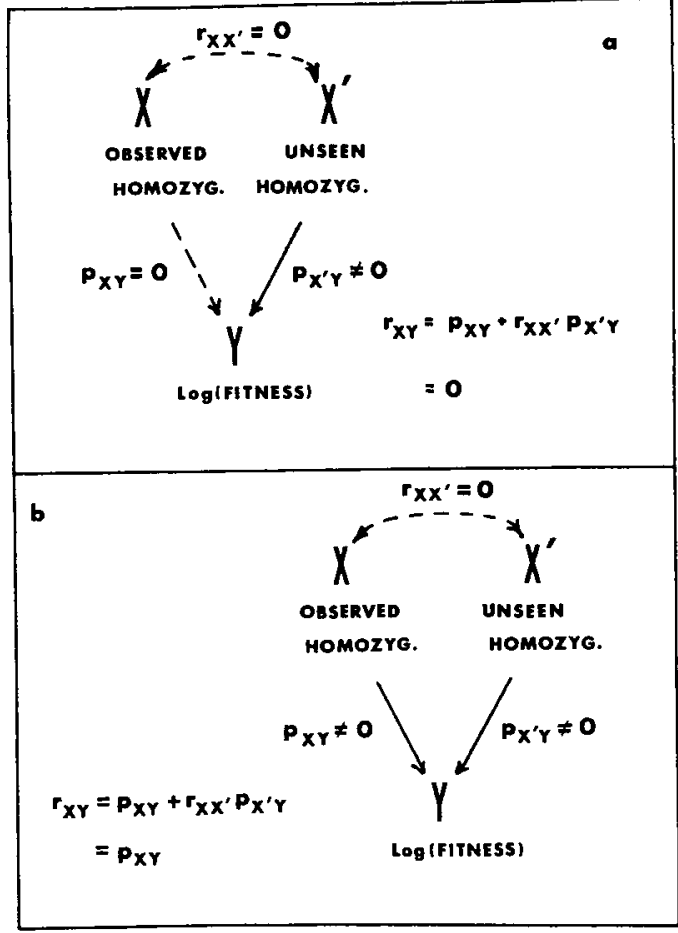

FIG. 4. Direct and indirect effects of observed homozygosity $(X)$ on $Y=\log (W)$ : a) the case where $p_{\mathrm{XY}}=0$ and $p_{\mathrm{X} Y} \neq 0$, and b) the case where $p_{\mathrm{XY}} \neq 0$ and $p_{\mathrm{X} \cdot \mathrm{Y}} \neq 0$.

adaptive distance $(X)$ and $(Y)=\log (W)$ is the theoretical reward. My assumption that $\exp \{-S\} \approx(1-S)$ and $\exp \{-T\} \approx(1-$ $T$ is tantamount to saying that there is no real distinction between the additive overdominance and multiplicative overdominance models, and there are no changes in the basic conclusions for the additive model.

Either of these simple models is only an approximation to a general fitness scheme for multiple unlinked loci, but the approximation may not be unreasonable as a first approximation. If marginal selective differentials are small at equilibrium, as seems empirically to be the case in most studies, it should be possible to approximate the $\log$ (fitness) values of particular multiplelocus genotypes by means of a Taylor expansion in the mathematical vicinity of equilibrium. In the parlance of Figure 1, the $Y$-value for $A_{1} A_{1} B_{1} B_{1}$ (for example) can be represented as: 


$$
\begin{aligned}
Y\left(A_{1} A_{1} B_{1} B_{1}\right) \approx 0-S_{\mathrm{A}} & -S_{\mathrm{B}} \\
& +O\left(S_{\mathrm{A}}+S_{\mathrm{B}}\right)^{2} .
\end{aligned}
$$

With $0<S_{\mathrm{A}}, S_{\mathrm{B}}<0.10$, the higher order terms should be very small. Multiplicative (additive) overdominance is surely not the true model, but within the limits of detection, it should be a reasonable first approximation. The regression strategy can be used to evaluate quadratic, cubic, or higher order (epistatic) models, of course, but with very little hope of a convincing demonstration, given small $S_{\mathrm{A}}$ and $S_{\mathrm{B}}$.

\section{ACKNOWLEDGMENTS}

I would like to thank Michael Boehnke, Robin Bush, Ranajit Chakraborty, Warren Ewens, Steve Frank, Tom Ledig, Jeffrey Long, Jeffry Mitton, Patricia Moll, James Neel, Pekka Pamilo, Clay Stephens, Hidenori Tachida, and an anonymous reviewer for many helpful comments on the manuscript. This work was supported by the following grants: NIH-GM-32589 and DoE84-ER60089.

\section{Literature Cited}

Bottini, E., F. Gloria-Bottini, P. Lucarelli, A. Polzonetti, F. Santro, ANd A. VARveri. 1979. Genetic polymorphisms and intrauterine development; evidence of decreasing heterozygosity in light-for-dates human newborn babies. Experientia 35:1565-1566.

Burton, R. S., and M. W. Feldman. 1983. Physiological effects of an allozyme polymorphism: Glutamate-pyruvate transaminase and response to hyperosmotic stress in the copepod Tigriopus californicus. Bioch. Genet. 21:239-251.

Cavener, D. R., And M. T. ClegG. 1981a. Multigenic response to ethanol in Drosophila melanogaster. Evolution 35:1-10.

- $1981 \mathrm{~b}$. Evidence for biochemical and physiological differences between enzyme genotypes in Drosophila melanogaster. Proc. Nat. Acad. Sci. USA 78:4444-4447.

Chakraborty, R. 1981. The distribution of the number of heterozygous loci in an individual in natural populations. Genetics 98:461-466.

Chakraborty, R., AND D. C. RaO. 1972. Detection of the inbreeding coefficient from $A B O$ blood group data. Amer. J. Hum. Genet. 24:352-354.

DiMichele, L., AND D. A. Powers. 1982. Physiological basis for swimming endurance differences between LDH-B genotypes of Fundulus heteroclitus. Science 216:1014-1016.

Ewens, W. J., And G. Thomson. 1977. Properties of equilibria in multi-locus genetic systems. Genetics 87:807-819.

Franklin, E. C. 1970. Survey of mutant forms and inbreeding depression in species of the family Pinaceae. U.S.D.A. For. Serv. Res. Pap. SE-61. Southeastern Forest Experiment Station, Asheville, NC.
Gaines, M. R., L. R. McClenaghan, JR., And R. K. ROSE. 1978. Temporal patterns of allozymic variation in fluctuating populations of Microtus ochrogaster. Evolution 32:723-739.

HABER, M. 1980. Detection of inbreeding effects by the $\chi^{2}$ test on genotypic and phenotypic frequencies. Amer. J. Hum. Genet. 32:754-760.

Hastings, A. 1981. Marginal underdominance at a stable equilibrium. Proc. Nat. Acad. Sci. USA 78: 6558-6559.

- 1982. Unexpected behavior in two locus genetic systems: An analysis of marginal underdominance at a stable equilibrium. Genetics 102:129138.

Hilbish, T. J., L. E. Deaton, and R. K. Koehn. 1982. Effect of an allozyme polymorphism on regulation of cell volume. Nature 298:688-689.

Karlin, S., and U. Liberman. 1979a. Central equilibria in multilocus systems I: Generalized nonepistatic selection regimes. Genetics 91:777-798.

- 1979b. Central equilibria in multilocus systems II: Bisexual generalized nonepistatic selection models. Genetics 91:799-816.

KoEHn, R. K., AND S. A. Shumway. 1982. A genetic/ physiological explanation for differential growth rate among individuals of the American oyster, Crassostrea virginica (Gmelin). Mar. Biol. Letters 3:3542.

Kojima, K., AND R. C. Lewontin. 1969. Evolutionary significance of linkage and epistasis, pp. $367-$ 388. In K. Kojima (ed.), Mathematical Topics in Population Genetics. Springer-Verlag, Berlin, W. Ger.

Knowles, P., And M. C. Grant. 1981. Genetic patterns associated with growth variability in ponderosa pine. Amer. J. Bot. 68:942-946.

Leary, R. F., F. W. Allendorf, ANd K. L. KNUdSEn. 1984. Superior developmental stability of heterozygotes at enzyme loci in Salmonid fishes. Amer. Natur. 124:540-551.

Ledig, F. T., R. P. Guries, AND B. A. Bonefeld. 1983. The relation of growth to heterozygosity in forest trees. Evolution 37:1227-1238.

Lewontin, R. C., And C. C. Cockerham. 1959. The goodness-of-fit test for detecting natural selection in random mating populations. Evolution 13:561564.

Lewontin, R. C., And K. Kojima. 1960. The evolutionary dynamics of complex polymorphisms. Evolution 14:458-472.

Lewontin, R. C., L. R. Ginzburg, and S. D. TUlJaPURKAR. 1978. Heterosis as an explanation for large amounts of genic polymorphism. Genetics 88:149-170.

Lı, C. C. 1978. First Course in Population Genetics. Boxwood Press, Pacific Grove, CA.

MANDEL, S. P. H. 1970. The equivalence of different sets of stability conditions for multiple allelic systems. Biometrics 26:840-845.

Mitton, J. B., And M. C. Grant. 1984. Association among heterozygosity, growth rate and developmental homeostasis. Ann. Rev. Ecol. Syst. 15:479499.

Mitron, J. B., And B. A. Pierce. 1980. The distribution of individual heterozygosity in natural populations. Genetics 95:1043-1054. 
Mitton, J. B., P. Knowles, K. B. Sturgeon, Y. B. LiNhART, AND M. DAvis. 1981. Association between heterozygosity and growth rate variables in western forest trees, pp. 27-34. In M. C. Conkle (ed.), Proceedings of the Symposium on Isozymes of North American Forest Trees and Forest Insects. U.S.D.A. For. Serv. Gen. Tech. Rep. PSW-48. Pacific Southwest Forest Experiment Station, Berkeley, CA.

Morton, N. E., J. F. Crow, ANd H. J. Muller. 1956. An estimate of the mutational damage in man from data on consanguineous marriages. Proc. Nat. Acad. Sci. USA 42:855-863.

Mukai, T., T. K. Watanabe, and O. Yamaguchi. 1974. The genetic structure of natural populations of Drosophila melanogaster. XII. Linkage disequilibrium in a large local population. Genetics 77: 771-793.

OAKeshott, J. G., J. B. Gibson, P. R. Anderson, W. R. Knibi, D. G. Anderson, and G. K. Chambers. 1982. Alcohol dehydrogenase and glycerol-3phosphate dehydrogenase clines in Drosophila melanogaster on different continents. Evolution 36:8696.

Pierce, B. A., AND J. B. Mitton. 1982. Allozyme heterozygosity and growth in the tiger salamander, Ambystoma tigrinum. J. Hered. 73:250-253.

SCHAAL, B. A., AND D. A. Levin. 1976. The demographic genetics of Liatris cylindracea Michx. (Compositae). Amer. Natur. 110:191-206.

SiNGH, S. M., AND E. Zouros. 1978. Genetic variation associated with growth rate in the American oyster (Crassostrea virginica). Evolution 32:342353.

TSAKAS, S., AND C. B. Krimbas. 1970. The genetics of Dacus oleae. IV. Relation between adult esterase genotypes and survival to organophosphate insecticides. Evolution 24:807-815.

Turelli, M., AND L. Ginzburg. 1983. Should individual fitness increase with heterozygosity? Genetics 104:191-209.

Vigue, C . L., And F. M. Johnson. 1973. Isozyme variability in species of the genus Drosophila. VI. Frequency-property-environment relationships of allelic alcohol dehydrogenases in $D$. melanogaster. Bioch. Genet. 9:213-227.

WARD, R. H., AND C. F. SING. 1970. A consideration of the power of the $\chi^{2}$ test to detect inbreeding effects in natural populations. Amer. Natur. 104: 355-366.

WATT, W. B. 1983. Adaptation at specific loci. II. Demographic and biochemical elements in the maintenance of the Colias PGI polymorphism. Genetics 103:691-724.

Watt, W. B., R. C. Cassin, And M. S. Swan. 1983. Adaptation at specific loci. III. Field behavior and survivorship differences among Colias PGI genotypes are predictable from in vitro biochemistry. Genetics 103:725-739.

Zouros, E., S. M. Singh, AND H. E. Miles. 1980. Growth rate in oysters: an over-dominant phenotype and its possible explanations. Evolution 34: 856-867.

Corresponding Editor: M. K. Uyenoyama

\section{APPENDIX}

The purpose of this appendix is to derive the expected means, sums of squares, and cross products needed for the regression analysis for a pair of unlinked, two-allele loci with multiplicative fitness values. Assume that the population is at two-locus Hardy-Weinberg equilibrium and that the allele frequencies are known without error. Using the definitions in the text, it develops that the expected value of $\bar{Y}$, the mean of the $Y_{j}$, is (since $\bar{\epsilon}=0$ ):

$$
\begin{aligned}
E(\bar{Y})= & P_{\mathrm{A}}^{2} P_{\mathrm{B}}^{2}\left(-S_{\mathrm{A}}-S_{\mathrm{B}}\right)+\ldots \\
& +Q_{\mathrm{A}}^{2} Q_{\mathrm{B}}^{2}\left(-T_{\mathrm{A}}-T_{\mathrm{B}}\right)+\bar{\epsilon} \\
= & -(\alpha+\beta),
\end{aligned}
$$

since at equilibrium:

$$
\begin{aligned}
P_{\mathrm{A}} & =\frac{T_{\mathrm{A}}}{S_{\mathrm{A}}+T_{\mathrm{A}}} \text { and } Q_{\mathrm{A}}=\frac{S_{\mathrm{A}}}{S_{\mathrm{A}}+T_{\mathrm{A}}} \\
P_{\mathrm{B}} & =\frac{T_{\mathrm{B}}}{S_{\mathrm{B}}+T_{\mathrm{B}}} \text { and } Q_{\mathrm{B}}=\frac{S_{\mathrm{B}}}{S_{\mathrm{B}}+T_{\mathrm{B}}} .
\end{aligned}
$$

The regression of $Y$ on $X_{\mathrm{A}}$ and $X_{\mathrm{B}}$ requires:

$$
\begin{aligned}
E[\mathrm{SS}(\mathrm{TOTAL})]= & E\left[\mathbf{Y}^{\prime} \mathbf{Y}\right] \\
= & E\left[\sum_{j=1}^{N}\left(Y_{j}-\bar{Y}\right)^{2}\right] \\
= & P_{\mathrm{A}}{ }^{2} P_{\mathrm{B}}{ }^{2} \cdot E\left[-S_{\mathrm{A}}-S_{\mathrm{B}}-\bar{Y}\right]^{2}+\ldots \\
& +Q_{\mathrm{A}}{ }^{2} Q_{\mathrm{B}}{ }^{2} \cdot E\left[-T_{\mathrm{A}}-T_{\mathrm{B}}-\bar{Y}\right]^{2} \\
& +E\left[\sum_{j=1}^{N} \epsilon_{j}^{2}\right] \\
= & (N-1) \cdot \sigma_{\mathrm{s}}{ }^{2}+(N-1)\left(\alpha^{2}+\beta^{2}\right) .
\end{aligned}
$$

Similar manipulations yield:

$$
\begin{aligned}
\mathbf{X}_{\mathrm{A}} \mathbf{X}_{\mathrm{A}} & =\sum_{j=1}^{N}\left(X_{\mathrm{A} j}-\bar{X}_{\mathrm{A}}\right)^{2} \\
& =(N-1) \\
& =\sum_{j=1}^{N}\left(X_{\mathrm{B} j}-\bar{X}_{\mathrm{B}}\right)^{2} \\
& =\mathbf{X}_{\mathrm{B}}{ }^{\prime} \mathbf{X}_{\mathrm{B}} \\
\mathbf{X}_{\mathrm{A}} \mathbf{X}_{\mathrm{B}} & =\sum_{j=1}^{N}\left(X_{\mathrm{A} j}-\bar{X}_{\mathrm{A}}\right)\left(X_{\mathrm{B} j}-\bar{X}_{\mathrm{B}}\right) \\
& =\mathbf{X}_{\mathrm{B}} \mathbf{X}_{\mathrm{A}} \\
& =0,
\end{aligned}
$$

and:

$$
\begin{aligned}
E\left[\mathbf{X}_{\mathrm{A}}{ }^{\prime} \mathbf{Y}\right] & =E\left[\sum_{j=1}^{N}\left(X_{\mathrm{A} j}-\bar{X}_{\mathrm{A}}\right)\left(Y_{j}-\bar{Y}\right)\right] \\
& =(N-1) \cdot \alpha \\
E\left[\mathbf{X}_{\mathrm{B}}{ }^{\prime} \mathbf{Y}\right] & =E\left[\sum_{j=1}^{N}\left(X_{\mathrm{B} j}-\bar{X}_{\mathrm{B}}\right)\left(Y_{j}-\bar{Y}\right)\right] \\
& =(N-1) \cdot \beta .
\end{aligned}
$$

The expected parameter estimates are: 


$$
\begin{aligned}
E[\hat{\theta}] & =\left(\mathbf{X}^{\prime} \mathbf{X}\right)^{-1} E\left(\mathbf{X}^{\prime} \mathbf{Y}\right) \\
& =\left[\begin{array}{ll}
\mathbf{X}_{\mathrm{A}}^{\prime} \mathbf{X}_{\mathrm{A}} & \mathbf{X}_{\mathrm{A}}^{\prime} \mathbf{X}_{\mathrm{B}} \\
\mathbf{X}_{\mathrm{B}}^{\prime} \mathbf{X}_{\mathrm{A}} & \mathbf{X}_{\mathrm{B}}^{\prime} \mathbf{X}_{\mathrm{B}}
\end{array}\right]^{-1} \cdot E\left[\begin{array}{l}
\mathbf{X}_{\mathrm{A}}^{\prime} \mathbf{Y} \\
\mathbf{X}_{\mathbf{B}}^{\prime} \mathbf{Y}
\end{array}\right] \\
& =\left[\begin{array}{l}
\alpha \\
\beta
\end{array}\right] .
\end{aligned}
$$

The expected model and error sums of squares are:

$E[\mathrm{SS}(\mathrm{MODEL})]=E\left[\left(\mathbf{Y}^{\prime} \mathbf{X}\right)\left(\mathbf{X}^{\prime} \mathbf{X}\right)^{-1} \cdot\left(\mathbf{X}^{\prime} \mathbf{Y}\right)\right]$

$$
=2 \cdot \sigma_{\mathrm{\varepsilon}}^{2}+(N-1) \cdot\left(\alpha^{2}+\beta^{2}\right) \quad \text { (A.7a) }
$$

$E[\mathrm{SS}(\mathrm{ERROR})]=E\left[\mathbf{Y}^{\prime}\left\{\mathbf{I}-\mathbf{X}\left(\mathbf{X}^{\prime} \mathbf{X}\right)^{-1} \mathbf{X}^{\prime}\right\} \mathbf{Y}\right]$

$$
=(N-3) \cdot \sigma_{t}^{2} \text {. }
$$

